\title{
Embedded Touchpad for Desktop Users
}

\author{
Parag J. Mondhe \\ Assistant Professor, \\ Department of Electronics \& \\ Telecomm Engg, \\ K. K. Wagh Institute of \\ Engineering Education \& Research, \\ Nashik.
}

\author{
Dinesh M. Chandwadkar, PhD \\ Professor \& Head, \\ Department of Electronics \& Telecomm Engg, \\ K. K. Wagh Institute of \\ Engineering Education \& Research, \\ Nashik.
}

\begin{abstract}
Few industries have changed so much in such a short time as the computer industry, and the pace of change shows no sign of slowing. The computer now has a role in almost every aspect of modern life, and it has radically affected the way people organize their lives. Desktop users have to push a mouse around their desktop while laptop users can just point a finger on touchpad. Touchpad is a type of computer input device which uses a touch sensitive surface to allow users to control the movement of a cursor, thus replacing a mouse, pointing stick or trackball. Instead of requiring a user to grasp a mouse and pivot at the wrist to click and track on the desktop, touchpads require less motion and allow users to situate their hands and arms in a variety of positions which results in less stress on wrists. Another major advantage of touchpads is that they take up less space than a traditional mouse so it is ideal where space is at premium. Therefore, many users and computer makers see touchpads as a viable, long-term alternative to other pointing devices. Therefore to benefit desktop users, the low cost embedded touchpad is proposed. It uses 4 wires resistive touchscreen to sense the position of a finger. The other hardware includes touchscreen controller circuit, AT89S52 microcontroller, MCP3208 ADC and MAX232 voltage converter. The software is written in Microsoft Visual Basic to operate curser on the screen.
\end{abstract}

\section{General Terms}

Embedded systems

\section{Keywords}

desktop users, embedded touchpad, pointing devices, 4 wires resistive touchscreen

\section{INTRODUCTION}

Throughout the past century, technology has improved in many ways. Globalization has led to wide use of computers as people across the world can easily connect among themselves. Computers have become a part and parcel of people's lives. On tip of the finger, we want all the work to be completed. Laptop computers generally use touchpad as an input device. A touchpad is a pointing device featuring a tactile sensor, a specialized surface that can translate the motion and position of a user's fingers to a relative position on screen. Touchpads are a common feature of laptop computers, and are also used as a substitute for a mouse where desk space is scarce because it relives the space required for mouse and mouse pad. Because they vary in size, they can also be found on personal digital assistants and some portable media players.

While using conventional mouse we have to keep our wrist in a slightly upward position all the time, due to the shape and volume of the mouse which result in stress on wrists. In contrast, with a touchpad we could leave our hand lying flat or hold it in whatever position we like. Touchpads can also be located directly in front of the user, such as below a keyboard. This prevents the need to reach off to one side to control a mouse, and also gives the option of controlling the touchpad with both hands. Therefore from ergonomics point of view, touchpad is more user friendly than the conventional mouse for long-term use [1].

\section{SYSTEM ARCHITECTURE}

The system architecture of proposed system is shown in figure 1. The 4 wires analog resistive touchscreen model HT043ANCNBD52-R developed by Han Touch is used in this system. Its output voltage indicates position of either $\mathrm{X}$ or $\mathrm{Y}$ intersection caused by touch at a time. To read value of $X$ position, $+5 \mathrm{~V}$ must be applied to pin 4 and pin 2 must be grounded. Then the output voltage is available on pin 3 [2]. The output voltage for $\mathrm{Y}$ position is available on pin 4 when $+5 \mathrm{~V}$ is applied on pin 1 and pin 3 is grounded. The touchscreen controller circuit is designed to take care of this operation as every pin of touchscreen has different function when reading output voltage for $\mathrm{X}$ and $\mathrm{Y}$ position.

The output voltage of touchscreen is analog in nature, which is converted to digital form using MCP3208 ADC IC. Two channels of ADC are used, one for each position [3]. The controller alternatively reads value for $\mathrm{X}$ and $\mathrm{Y}$ position and sends it to desktop computer using RS 232 serial communication standard. Two push buttons are used to perform function of left and right click. These push buttons are directly interfaced to microcontroller. When any of them is pressed by user, microcontroller sends predefined code to the computer. The software written in Microsoft Visual Basic performs the task of operating cursor on the screen. The entire system works on $+5 \mathrm{~V}$ power supply.

\section{HARDWARE DESIGN \\ 3.1 4 Wires Resistive Touchscreen}

A touchscreen is two dimensional sensing device that is constructed of two sheets of material separated slightly by spacers. A glass sheet is used to provide stable bottom layer and Polyethylene (PET) sheet is used as a flexible top layer [2]. The spacers may have opening to allow venting. The two sheets are coated with resistive substance, a metal compound called Indium Tin Oxide (ITO).

The ITO is thinly and uniformly sputtered onto both the glass and PET layer. Tiny bumps called spacer dots are then added to the glass side, on top of resistive ITO coating, to keep the PET film from sagging, causing an accidental or false touch. 


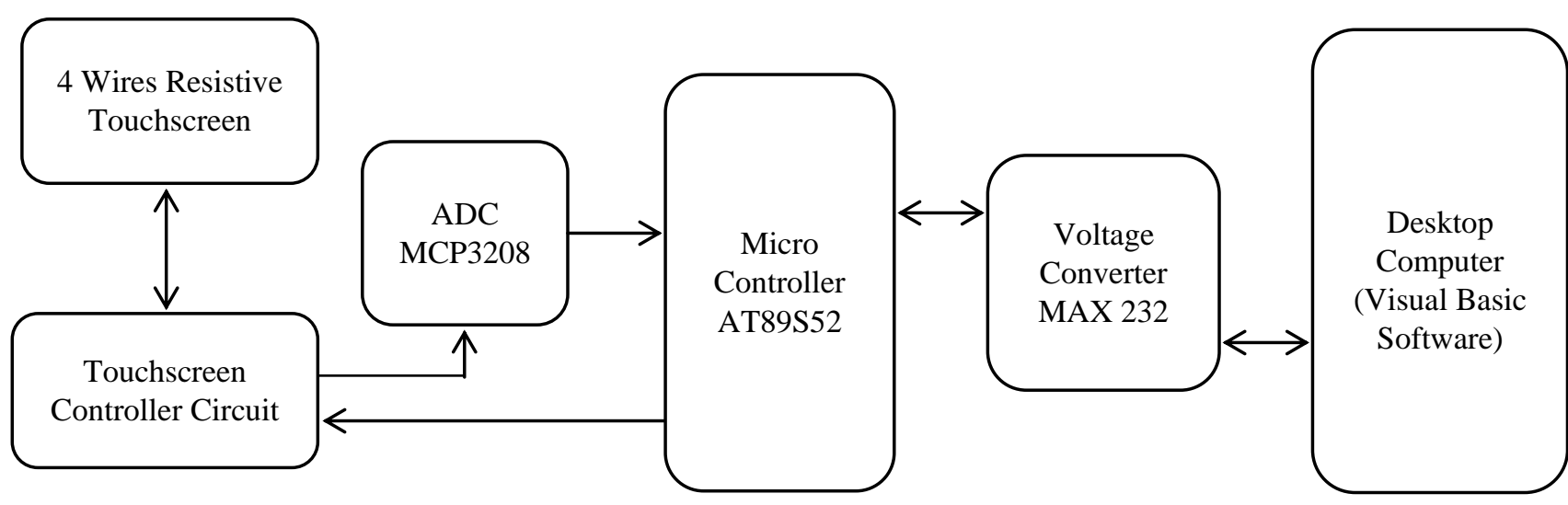

Figure 1: System architecture

The amount of pressure needed to cause touch event is largely determined by the size and spacing of the spacer dots [4]. The bus bars and traces, made up of conductive silver ink, connect the ITO layer to flex tail. Silver ink is also used to connect top traces to bottom traces. When the PET film is pressed down the two surfaces meet. The position of this meeting (a touch) can be read by touch screen controller circuit. The resistance is from 400 to $1200 \mathrm{ohm}$ for $\mathrm{X}$ position while it is from 100 to $700 \mathrm{ohm}$ for Y position [2].

\subsection{Touchscreen Controller Circuit}

Touchscreen controller circuit is designed to switch function of each pin when reading X and Y position [5]. Two NPN and two PNP transistors together with some resistors are used to form this circuit. These transistors are used as a switch and operate in either saturation or cut off region. Base terminal of these transistors is connected through resistor to the port pin of the microcontroller. Microcontroller turned these transistors on or off independently according to the table 1 for position to be read.

\subsection{AT89S52 Microcontroller}

The AT89S52 a 40 pin, low-power, high-performance 8 bit microcontroller is sufficient for this application. The AT89S52 provides $8 \mathrm{~K}$ bytes of in-system programmable flash, 256 bytes of RAM, 32 I/O lines, Watchdog timer, two data pointers, three 16-bit timer/counters, a six-vector twolevel interrupt architecture, a full duplex serial port, on-chip oscillator, and clock circuitry [6]. In addition, the AT89S52 also supports two software selectable power saving modes.

\subsection{MCP3208 ADC}

The To provide higher resolution 12 bit analog to digital converter developed by Microchip MCP3208 is used. It features successive approximation technique and has 8 single ended input channels. It has 16 pins and its sampling rate is $100 \mathrm{k}$ samples per second [7]. It combines high performance and low power consumption in a small package, making it ideal for embedded applications.

\subsection{MAX232 Voltage Converter}

To match TTL and RS 232 voltage levels, MAX232 IC is used as a voltage converter [8]. It has 16 pins and operates on
$+5 \mathrm{~V}$. It consists of voltage doubler, voltage inverter and 2 pairs of voltage transceivers [9]. TXD and RXD pins of microcontroller are used for serial communication. DB 9 connector is used for connection with serial port of a computer.

\section{SOFTWARE DESIGN}

\subsection{Microcontroller Programming}

The program for microcontroller is written using assembly language. After initialization the microcontroller sends signals to touchscreen controller circuit to read position X. Then it sends start of conversion signal to ADC. Microcontroller waits for an end of conversion signal from ADC. After receiving that signal, it reads output value from $\mathrm{ADC}$ and stores it in memory.

Microcontroller follows similar steps to store value of $\mathrm{Y}$ position. Then it checks whether switch provided for left or right click is pressed. Microcontroller combines the entire data in predefined format and sends it to computer using RS232 serial communication standard. It follows this sequence of operation every time.

\subsection{Microsoft Visual Basic}

Microsoft Visual Basic is a third-generation event driven programming language and integrated development environment from Microsoft. Visual Basic was derived from BASIC and enables the rapid application development of graphical user interface applications, access to databases using Data Access Objects, Remote Data Objects, or ActiveX Data Objects, and creation of ActiveX controls and objects.

An application can be developed using the components provided with Visual Basic. Programs written in Visual Basic can also use the Windows API, by declaring external functions. Programming in VB is a combination of visually arranging components or controls on a form, specifying attributes and actions of those components, and writing additional lines of code for more functionality [10]. Since default attributes and actions are defined for the components, a simple program can be created without writing many lines of code.

Table 1. Pin functions of touchscreen

\begin{tabular}{|c|c|c|c|c|}
\hline Pin No: & $\mathbf{1}$ & $\mathbf{2}$ & $\mathbf{3}$ & $\mathbf{4}$ \\
\hline X position & Don't care & $0 \mathrm{~V}$ & Output Voltage & $+5 \mathrm{~V}$ \\
\hline Y position & $+5 \mathrm{~V}$ & Don't care & $0 \mathrm{~V}$ & Output Voltage \\
\hline
\end{tabular}


Table 2. Comparison of various touchscreen technologies

\begin{tabular}{|l|l|l|l|l|}
\hline Parameters & Resistive & Capacitive & Infrared & Surface acoustic \\
\hline Technology & Surface Technique & Surface Technique \\
$($ Electrical $)$ & Electrical $)$ & $\begin{array}{l}\text { Edge Technique } \\
(\text { Optical })\end{array}$ & $\begin{array}{l}\text { Surface Technique } \\
\text { (Acoustic) }\end{array}$ \\
\hline Transparency & Bad & Normal & Good & Good \\
\hline Durability & High & Normal & High & Higher \\
\hline Speed & Higher & High & High & Highest \\
\hline Cost & Lowest & Moderate & High & High \\
\hline
\end{tabular}

\subsection{Microsoft Visual Basic Software}

The software to control operation of cursor on screen is written using Microsoft Visual Basic. The software read the data send by the microcontroller from the serial port selected by user, when the user presses Connect button in the window of software to enable touchpad functioning. The data sent by microcontroller is in TXXXYYYLR format. $\mathrm{T}$ is used for identification purpose. The XXX indicates $\mathrm{X}$ position while YYY indicates $Y$ position of touch on touchscreen.

Microcontroller will send $\mathrm{P}$ when the left button is pressed at the position of $\mathrm{L}$ otherwise $\mathrm{N}$ will be send. Same convention will be followed for $\mathrm{R}$ when right button is pressed. Left or right click operation is performed if the read data shows that respective key is pressed. The $\mathrm{X}$ and $\mathrm{Y}$ position value is retrieved from the read data. This value is compared with the threshold to filter noise. The current values for $\mathrm{X}$ and $\mathrm{Y}$ position are calculated from subtracting them from previous values. Initially previous values are set to zero. The cursor is moved to the new values of $\mathrm{X}$ and $\mathrm{Y}$ position. The software repeats all this procedure continuously with very high speed.

\section{RESULTS AND FUTURE SCOPE}

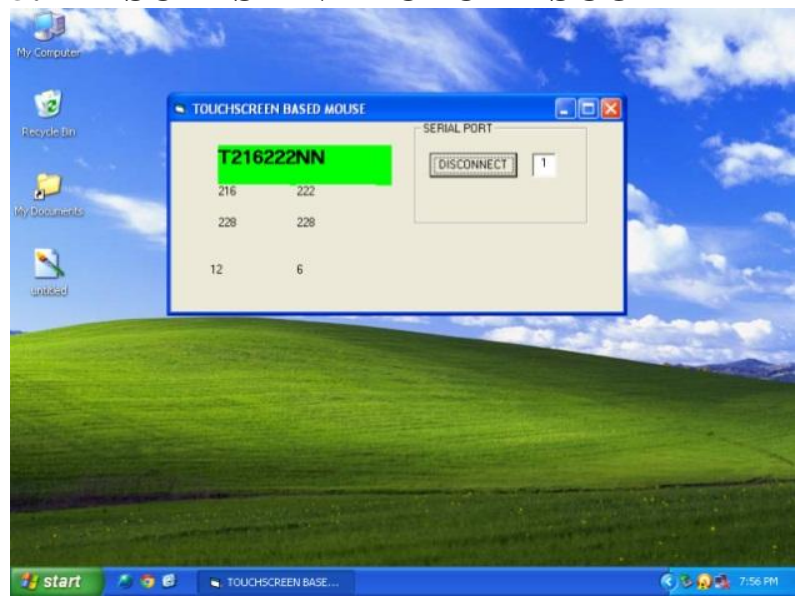

Figure 2: Screenshot when touchpad is working

One time installation of software on the desktop computer is required to use this embedded touchpad. The DB 9 connector can be connected to any of the serial port of the computer. As shown in figure 2, when Connect button is pressed and appropriate serial port is selected by user the touchpad starts functioning successfully. The software window shows current and previous values $\mathrm{X}$ and $\mathrm{Y}$ positions along with difference between them. This touchpad is able to perform all the functions performed by the conventional mouse. To disable the functioning of the touchpad Disconnect button can be pressed.

Moving towards the future, consumers will continue to see the growth of the touchscreen industry, due to extensive engineering advancements in user interfaces. The ability to physically touch is easier than searching for a specific key in a sea of buttons. Society, for these reasons, has found touch screens to be the future of many devices.

The resistive touchscreen has better response time and long duration of operation compared to its counterpart such as capacitive or infrared touchscreens as table 2 shows. Resistive touchscreens are generally the most affordable type of touch screen, which explains their success in high use applications. The two most popular resistive architectures use 4 wires or 5 wires configuration. The 5 wires configuration has one extra wire for wiper contact of second layer.

A cost effective touchpad with simple hardware and software which will satisfy the needs of end user is implemented and working successfully. Various features can be added to this basic design, such as it can be made wireless using Bluetooth or other medium. To offer an enhanced level of control we can allow users to perform gestures with one or more fingers to give a series of commands such as scrolling, zooming and navigating between windows or Web pages. Using similar type of technology touch screen keypad can also be designed.

\section{REFERENCES}

[1] C. Forlines, D. Wigdor, C. Shen, R. Balakrishnan, 2007. Direct-Touch vs. Mouse Input for Tabletop Displays, CHI Proceedings, pp. 647-656

[2] Datasheet of 4 wire analog resistive touchscreen model HT043A-NCNBD52-R http://www.hantouchusa.com/4__wire.htm

[3] S. Pietri, A. Olmos, M. Berens, A. V. Boas, M. Goes, 2009. A fully integrated touch screen controller based on $12 \mathrm{~b} \quad 825 \mathrm{kS} / \mathrm{s}$ SAR ADC, Micro-Nano electronics Technology and Applications, pp. 66-70

[4] T. Hoye, J. Kozak, 2010. Touch screens: a pressing technology, Tenth annual freshman conference, pp. 1-6

[5] R. N. Aguilar, G. C. M. Meijer, 2002. Fast interface electronics for a resistive touch-screen. Sensors, Proceedings of IEEE, Volume 2, pp. 1360 - 1363

[6] Datasheet of http://www.atmel.com/Images/doc1919.pdf
AT89S52 
International Journal of Computer Applications (0975 - 8887)

Volume 163 - No 6, April 2017

[7] Datasheet

$$
\text { of }
$$

MCP3208

http://www.microchip.com/downloads/en/devicedoc/212 98c.pdf

[8] M. A. Mazidi, J. G. Mazidi and R. McKinlay, 2007. The 8051 Microcontroller and Embedded Systems Using Assembly and C, 2 edition, Pearson Education

[9] Datasheet of

MAX232

http://www.maximintegrated.com/datasheet/index.mvp/i
d/1798?ver=C\&utm_expid=50713806-

2\&utm_referrer=http $\% 3 \mathrm{~A} \% 2 \mathrm{~F} \% 2 \mathrm{Fwww}$.maximintegrate d.com $\% 2$ Fdatasheet $\% 2$ Findex.mvp $\% 2$ Fid $\% 2 \mathrm{~F} 1798 \% 3 \mathrm{~F}$ ver\%3DC

[10] E. Petroutsos, 2010. Mastering Microsoft Visual Basic 2010, Wiley India 